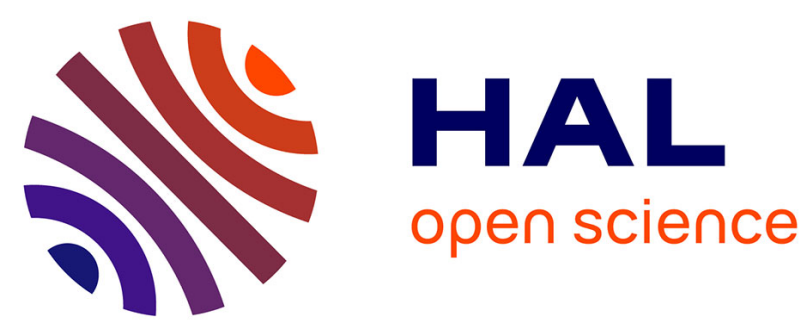

\title{
Photo-Thermal Switching of Individual Plasmonically Activated Spin Crossover Nanoparticle Imaged by Ultrafast Transmission Electron Microscopy
}

Yaowei Hu, Matthieu Picher, Ngoc Minh Tran, Marlène Palluel, Laurentiu Stoleriu, Nathalie Daro, Stephane Mornet, Cristian Enachescu, Eric Freysz, Florian Banhart, et al.

\section{To cite this version:}

Yaowei Hu, Matthieu Picher, Ngoc Minh Tran, Marlène Palluel, Laurentiu Stoleriu, et al.. PhotoThermal Switching of Individual Plasmonically Activated Spin Crossover Nanoparticle Imaged by Ultrafast Transmission Electron Microscopy. Advanced Materials, 2021, 33 (52), 2105586 (10 p.). 10.1002/adma.202105586 . hal-03367060

\section{HAL Id: hal-03367060 https://hal.science/hal-03367060}

Submitted on 6 Oct 2021

HAL is a multi-disciplinary open access archive for the deposit and dissemination of scientific research documents, whether they are published or not. The documents may come from teaching and research institutions in France or abroad, or from public or private research centers.
L'archive ouverte pluridisciplinaire HAL, est destinée au dépôt et à la diffusion de documents scientifiques de niveau recherche, publiés ou non, émanant des établissements d'enseignement et de recherche français ou étrangers, des laboratoires publics ou privés. 


\section{Photo-Thermal Switching of Individual Plasmonically Activated Spin Crossover Nanoparticle Imaged by Ultrafast Transmission Electron Microscopy}

Yaowei Hu, Matthieu Picher, Ngoc Minh Tran, Marlène Palluel, Laurentiu Stoleriu, Nathalie Daro, Stephane Mornet, Cristian Enachescu, Eric Freysz, Florian Banhart* and Guillaume Chastanet ${ }^{*}$

Y. Hu, Dr. M. Picher, Prof. F. Banhart

Institut de Physique et Chimie des Matériaux, UMR 7504, Université de Strasbourg, CNRS, F-67034 Strasbourg, France. E-mail : florian.banhart@ipcms.unistra.fr

Dr. N.M. Tran, Dr. E. Freysz

Univ. Bordeaux, CNRS, UMR 5798, LOMA, 358 Cours de la libération, F-33405

Talence cedex, France E-Mail: eric.freysz@u-bordeaux.fr

Dr. L. Stoleriu, Dr. C. Enachescu

Faculty of Physics, Alexandru Ioan Cuza University, 700506 Iasi, Romania

Dr. M. Palluel, Dr. N. Daro, Dr. S. Mornet, Dr. G. Chastanet

Institut de chimie de la Matière Condensée de Bordeaux, CNRS, Univ. Bordeaux, Bordeaux INP, UMR 5026, F-33600, Pessac, France. E-mail : guillaume.chastanet@icmcb.cnrs.fr

Keywords: ultrafast electron microscopy, single nanoparticle detection, spin crossover, timeresolved spectroscopy, nanohybrids

Abstract: Spin Crossover (SCO) is a promising switching phenomenon when implemented in electronic devices as molecules, thin films or nanoparticles. Among the properties modulated along this phenomenon, optically induced mechanical changes are of tremendous importance as they can work as fast light-induced mechanical switches or allow to investigate and control microstructural strains and fatigability. The development of characterization techniques probing 
nanoscopic behaviour with high spatio-temporal resolution allows to trigger and visualize such mechanical changes of individual nanoscopic objects. Here we use Ultrafast Transmission Electron Microscopy (UTEM) to precisely probe the length changes of individual switchable nanoparticles induced thermally by nanosecond laser pulses. This allows us to reveal the mechanisms of spin switching, leading to the macroscopic expansion of SCO materials. This study was conducted on individual pure SCO nanoparticles and SCO nanoparticles encapsulating gold nanorods that serve for plasmonic heating under laser pulses. Length changes are compared with time-resolved optical measurements performed on an assembly of these particles.

\section{Introduction}

Materials that enable mechanical actuation or sensing under stimulation are highly demanded for many applications. Among such materials, spin crossover (SCO) systems (mainly metallic coordination complexes $)^{[1,2,3]}$ are investigated because the stimulus-induced electronic configuration change between low spin (LS) and high spin (HS) states is accompanied by an average local volume change of the coordination polyhedron of the metal site experiencing the spin crossover of $25 \%$. In the crystals, this local volume change at the metal site propagates through the whole material by intermolecular interactions, producing up to around $10 \%$ expansion or contraction of the whole crystal ${ }^{[4]}$, thus making them of interest as actuators ${ }^{[5,6,7]}$ but also in electronic devices ${ }^{[8,9,10]}$ or in nanothermometry ${ }^{[11]}$. The ultrafast spin state switching deserves investigations on the impact of the mechanical changes at the nanoscale especially in opto-electronic $^{[12,13]}$ or memristance ${ }^{[14,15,16]}$ devices involving SCO. Indeed, this volume change is likely to create internal strains and therefore alter the SCO through structural fatigability $^{[17,18,19]}$, even if mechanical resilience ${ }^{[20]}$ has been evidenced. This point is of importance for device durability upon intensive use since a change in the microstructural features can affect the switching properties and consequently the reliability of the device. 
Spin crossover can be induced by several means like temperature, pressure and light irradiation. Optical switching of spin crossover materials is of great interest because of the large prospects for optical memories. The optical switching of these materials may be achieved through several processes: light irradiation can promote a photo-reaction on ligands, populate excited states, or heat the sample ${ }^{[21]}$. Previous studies on the LS $\rightarrow$ HS (respectively HS $\rightarrow$ LS) photo-excitation, also referred as Light-Induced Excited Spin State Trapping (LIESST, respectively reverse-LIESST), showed this is a multistep process that allows the population at low temperature of the metastable $\mathrm{HS}^{*}$ state $^{[22,23]}$ : (i) The LS to HS* state photoswitching occurs in few femtoseconds, with a local increase of the coordination sphere volume, but at constant unit-cell volume. (ii) These newly formed HS* molecules generate an internal pressure on the LS lattice while the energy deposited by the laser dissipates through lattice heating. These stresses are relaxed through dilation of the lattice mediated by acoustic phonons at time scales determined by the size of the crystals and the speed of sound in the material. (iii) This lattice expansion, which takes place few nanoseconds after the excitation, can promote the switching of additional molecules toward the HS* state, and increases the average crystal temperature as well as its volume. This increase of temperature gets faster as the crystal size is reduced. The whole phenomenon is then highly non-linear since one photon can convert several sites ${ }^{[24]}$.

As mentioned above, the temperature increase induced by the laser excitation can also be used to trigger the LS to HS spin crossover. Here we study the photo-thermal case, where the process is mainly limited by the diffusion of heat within the sample. This process strongly depends on the size of the particles ${ }^{[24]}$ and therefore only the steps occurring above the nanosecond time scale are at play. It is of strong interest when applied to cooperative SCO materials. In such materials, the strong intermolecular interactions ${ }^{[25]}$ involve an additional energy to overcome, leading to the occurrence of hysteretic SCO features. As a consequence, memory effects are accessible, around room temperature, for such systems. When the irradiation is applied onto the LS state, at a temperature inside the hysteresis, the laser pulse 
induces a quantitative LS $\rightarrow$ HS irreversible photoswitching due to the stable character of the HS state in the hysteresis ${ }^{[26,27,28,29,30,31,32,33,34,35]}$. This can be applied to generate room temperature opto-electronic devices ${ }^{[13]}$. The efficiency and speed of such photothermal effect can be highly improved by reducing the size of the particles ${ }^{[2,36]}$ and incorporating nanoheaters into the SCO particles, like gold nanoparticles that produce heat when excited in their surface plasmon resonance $(\mathrm{SPR})$ band $^{[37]}$. Indeed, the absorption coefficient of plasmonic nanoparticles is much higher than of the SCO compound. Therefore, excitation of the SPR band should heat the sample more efficiently compared to direct absorption of light by the SCO component. Strong reductions of the photothermal LS to HS switching threshold were observed while incorporating gold NPs onto (SCO@ Au architectures) or inside (Au @ SCO architectures) the SCO particles ${ }^{[38,39,40,41,42,43]}$.

For the application of SCO materials in opto-mechanical devices, it is indispensable to know the shape changes of individual nanoparticles and, in particular, the time-dependent response on photon pulses. In most previous studies, the response of large ensembles of SCO particles to laser pulses was studied at a macroscopic scale. However, these measurements did not reveal the behaviour of individual SCO particles and thus didn't allow to detect size changes of the nanocrystals. Indeed, the knowledge of the exact volume change of a single SCO particle, its time scale upon photo-switching and its repeatability is of importance to understand the transport mechanisms, the occurrence of percolation effect (when the increase of volume induces nanoparticles to be in contact) and the fatigue of the devices. A recent study has shown the thermal hysteresis loop and LS to HS switching kinetic at the second time scale on individual nanoparticles through optical methods without providing information on the volume change $^{44}$. In a previous investigation ${ }^{[45,46]}$, ultrafast TEM has already shown shape changes of SCO particles after laser irradiation, but details of this transformation and the influence of embedded plasmonically active particles remain unexplored. The knowledge of the photoswitching dynamic as function of the laser fluence, the efficiency of the heat transfer, the 
influence of the substrate bearing the nanoparticles, and other parameters are still to be investigated and remain a challenge. In this paper, time-resolved measurements of the switching dynamics are carried out on individual pure and gold-filled SCO nanoparticles by ultrafast electron microscopy. The expansion is measured under laser pulses of different energies at the nanosecond to the millisecond timescale. This study is performed on single nanoparticles and compared with an optical characterization of an assembly of particles. Such an investigation is of importance as it can be extended to breathing or mechanically active materials (photochromic materials, batteries upon charge and discharge, actuators, nanomachines...).

\section{Results and Discussion}

\subsection{Studied systems}

In this study, we focus our attention on triazole-based SCO nanoparticles which are widely studied for implementation in devices ${ }^{[5,8,13]}$. They are built from a $1 \mathrm{D}$ coordination polymer made of $\mathrm{Fe}(\mathrm{II})$ metal ionic switchable sites linked together by bridging triazole ligands to form linear chains separated by counter-anions ${ }^{[47]}$. The $\left[\mathrm{Fe}(\mathrm{Htrz})_{2} \operatorname{trz}\right]\left(\mathrm{BF}_{4}\right)$ is probably the most studied compound since large hysteresis loops can be observed above room temperature ${ }^{[48,49]}$ and at the nanoscale ${ }^{[36,50]}$. The photo-thermal effect on $115 \mathrm{~nm}$ nanoparticles of this compound has been reported to occur within 12 ns by optical measurements performed on an assembly of $\operatorname{particles}^{[32]}$.

Gold nanorods were embedded into these SCO nanoparticles to promote local heating through light-activation of the surface plasmon resonance ${ }^{[41]}$ (Figure 1a and supporting information for the protocol). An assembly of such nanohybrids exhibits a wide thermal hysteresis of $34 \mathrm{~K}$, centered at $360 \mathrm{~K}$ with switching temperatures in the heating mode $\mathrm{T}_{1 / 2} \uparrow=$ $378 \mathrm{~K}$ and in cooling mode $\mathrm{T}_{1 / 2} \downarrow=344 \mathrm{~K}$ (Figure 1b). Moreover, on the same synthetic batch, the SCO nanoparticles can host one or several gold nanorods. 


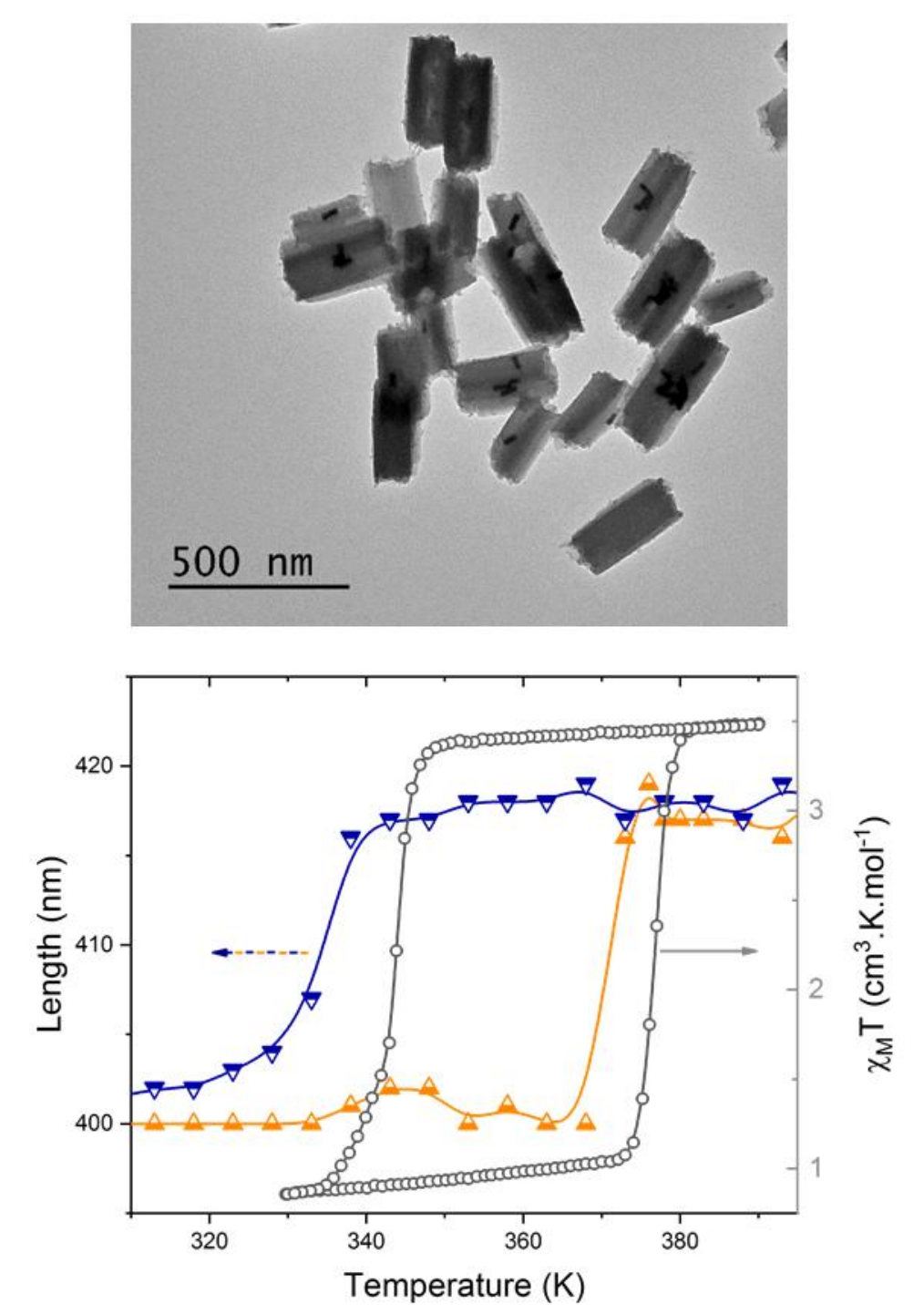

Figure 1: a) TEM image of the Au@SCO nanohybrids with various amounts of gold nanorods inside the SCO particles (from 0 to 7). b) Thermal dependence of the magnetic susceptibility (black, for an ensemble of particles) and length of a single pure SCO particle along its long axis (orange/blue). The length measurements were taken in the heating stage of a conventional TEM with continuous electron beam. The accuracy of the length measurements is approximately $1 \mathrm{~nm}$. A similar hysteresis taken from another SCO particle and a Au@SCO particle is shown in fig. SI2.

\subsection{Static temperature effects}


In previous studies, the crystal structure of the one-dimensional coordination polymer $\left[\mathrm{Fe}(\mathrm{Htrz})_{2} \operatorname{trz}\right]\left(\mathrm{BF}_{4}\right)$ showed changes in the unit-cell volume by $11 \%$ with a pronounced elongation of the $\mathrm{b}$ axis (axis along which the one dimensional polymer grows) of $0.46 \AA$ from LS to HS, corresponding to an expansion of $6 \%^{[51]}$. For a $400 \mathrm{~nm}$ nanoparticle, this should correspond to almost $24 \mathrm{~nm}$ of stretching.

In the first set of experiments, the static lengths of pure SCO particles are measured in a wide temperature range. This is carried out at thermal equilibrium by continuous heating of SCO particles in a high-temperature TEM specimen stage. The length changes are measured by taking conventional images with a continuous electron beam. Figure 1b shows TEM measurements of the expansion of a single pure SCO particle along its long axis together with the magnetic susceptibility of a macroscopic amount of SCO particles as a function of temperature. The same measurements for a Au@SCO particle are shown in Figure SI2. Both measurements show a hysteresis upon heating and cooling. While the hysteresis of the first cycle taken from a new specimen is always approximately $10 \mathrm{~K}$ wider, all following curves were precisely reproducible upon repeated heating-cooling cycles, in agreement with the known run-in process for this compound ${ }^{[17,48,49,50]}$. The hysteresis is approximately $37 \mathrm{~K}$ wide for both measurements; the switching temperatures of the length changes are in the warming mode $\mathrm{T}_{1 / 2} \uparrow$ $=371 \mathrm{~K}$ and in the cooling mode $\mathrm{T}_{1 / 2} \downarrow=334 \mathrm{~K}$. The switching temperatures measured through the length change are slightly shifted towards low temperature compared to the susceptibility measurements. This may arise from various sources, the most probable ones being the different temperature calibrations ( $+/-5^{\circ}$ in the UTEM heating stage) and a slight length difference (330 $\mathrm{nm}$ for the magnetic measurement versus $400 \mathrm{~nm}$ for UTEM measurements for about the same width). The hysteresis from a Au@SCO particle (Fig. SI2) is qualitatively similar but slightly narrower, most likely due to the non-expanding Au rods that replace some SCO material. The slightly varying length within the hysteresis appears in all measurements but isn't understood in detail yet. 
This measurement clearly shows that upon warming, the nanoparticle expands from $400 \mathrm{~nm}$ to $416 \mathrm{~nm}$. This corresponds to $5 \%$ of elongation, very close to the $6 \%$ increase in lattice parameter along the c-axis recorded by X-ray crystallography. The TEM observation shows that the widening is less pronounced than the lengthening, in accordance with the expected anisotropic expansion (which is larger for the $b$, than for $a(+0.9 \%)$ and $c(+4 \%)$ unit-cell parameters). These results confirm the observation of the bistable effect within the hysteresis loop on one individual nanoparticle of $330 \mathrm{~nm}$, in agreement with the report on $200 \mathrm{~nm}$ particles of the same compound ${ }^{[46]}$ recorded by surface plasmon resonance microscopy and on $600 \mathrm{~nm}$ particles of another SCO compound ${ }^{[45]}$ recorded by electron diffraction.

\subsection{Time-resolved TEM studies on individual nanoparticles}

The optical absorption of SCO at the wavelength of the laser pulses in this study (1064 nm) is very low and so allows us to study the heating effect of the embedded Au rods. Therefore, pure SCO particles are mainly heated from the carbon substrate in our setup ${ }^{[46]}$ and Au@SCO from the embedded Au rods ${ }^{[41]}$. In the first case, the copper grid holding the graphene support film on which the SCO particles are deposited absorbs the heat which then diffuses through the graphene layer to the SCO particles. In the second case, the encapsulated Au nanorods heat the SCO particles much more efficiently from inside. No static specimen heating was applied in the time-resolved studies.

Figure 2 shows UTEM images of a SCO particle (see Methods section and figure SI1) taken with nanosecond electron pulses before and $500 \mathrm{~ns}$ after an IR pulse $\left(1064 \mathrm{~nm}, 7 \mathrm{~ns}, 45 \mathrm{~mJ} \mathrm{~cm}^{-}\right.$ ${ }^{2}$ ). As seen from the contrast profiles along the long axis of the particle, the length has increased by $20 \mathrm{~nm}$ which corresponds to $5 \%$ of its total length. A width change of $3 \mathrm{~nm}$ corresponding 
to $2 \%$ of the total width was measured. This result is in accordance with the expected expansion anisotropy of the SCO particles. Here the $b$ axis corresponds to the long axis of the particles.
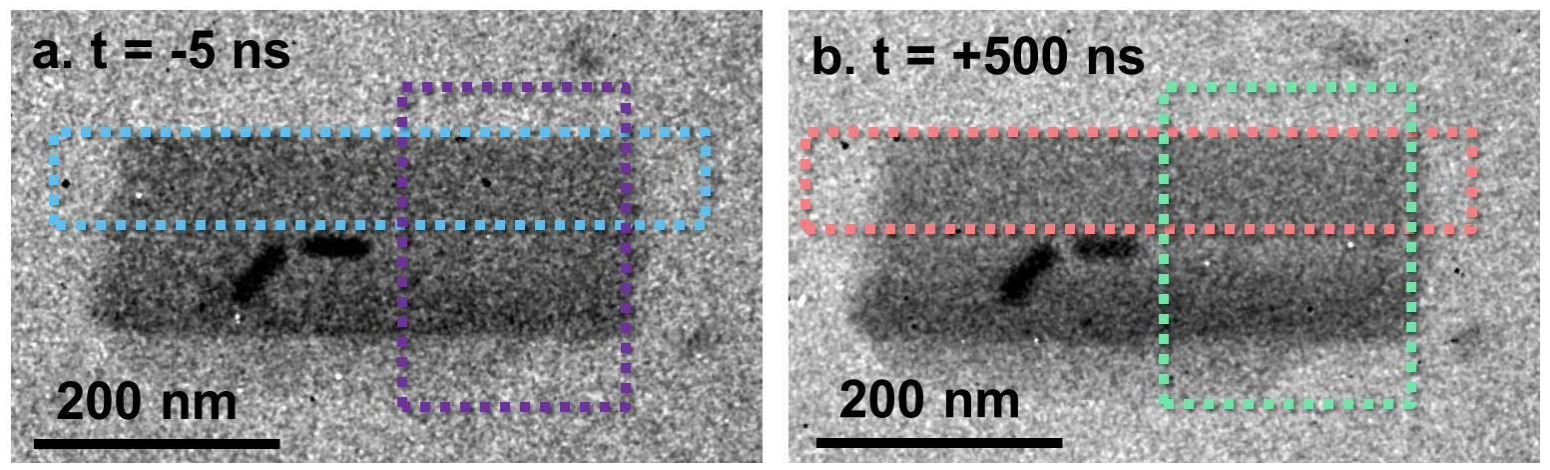

c.

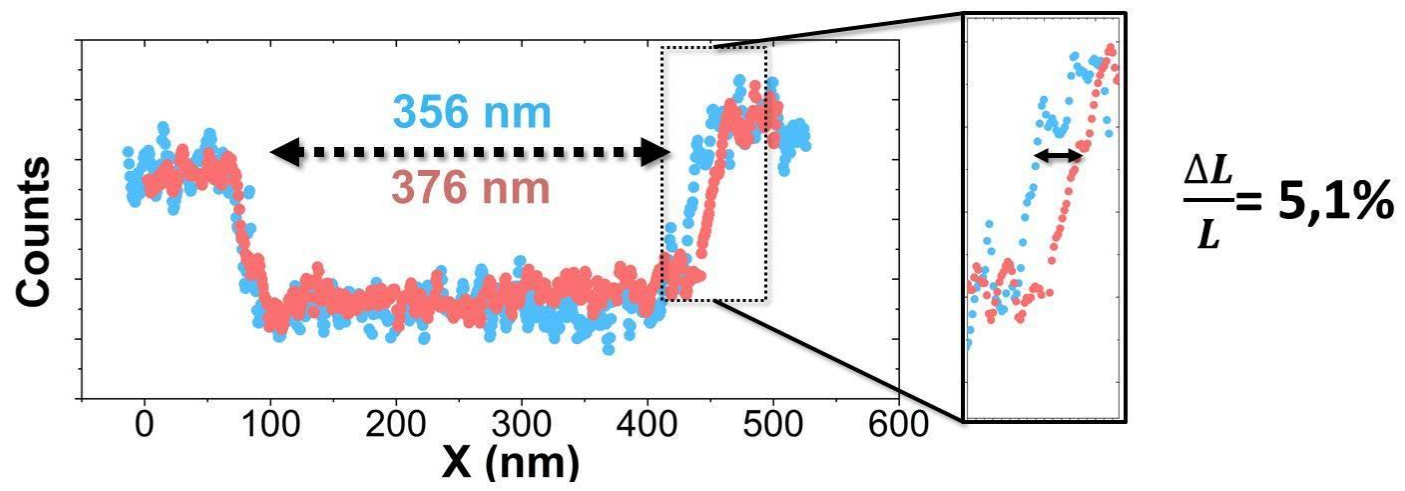

d.

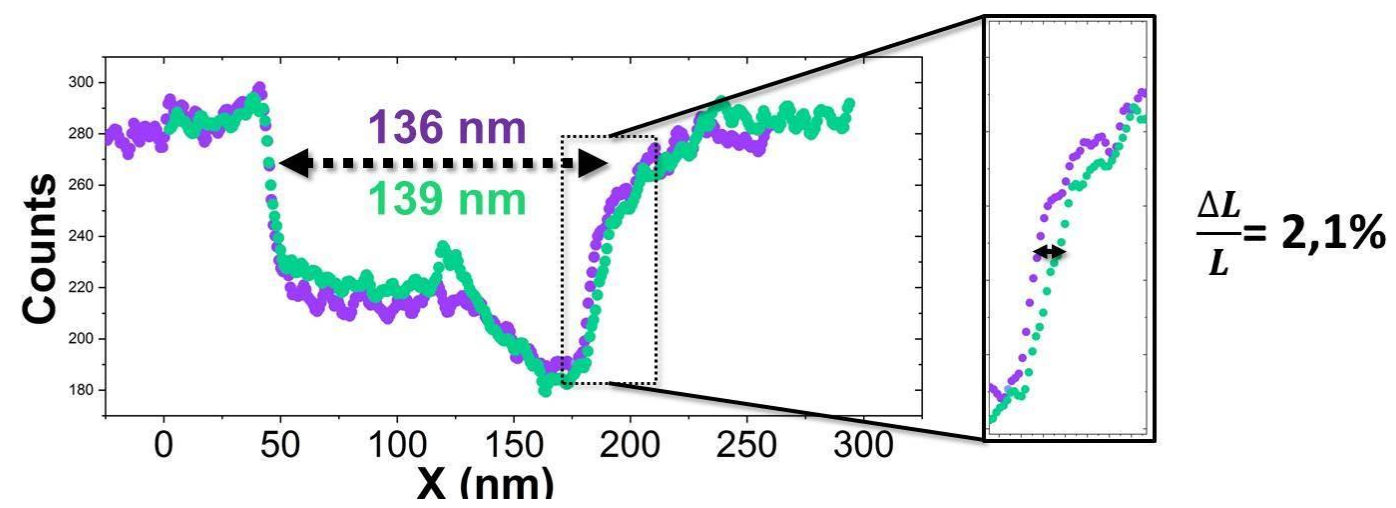

Figure 2: SCO particle encapsulating two Au nanorods before (a) and $500 \mathrm{~ns}$ after $(b)$ an IR laser pulse of $8 \mu \mathrm{J}$ focused onto a spot of $150 \mu \mathrm{m}$ in diameter. The profiles $(c, d)$ show the size change along the long and short axes.

Figure 3a shows the expansion of a pure (unfilled) SCO particle (LS length $390 \mathrm{~nm}$ ) as a function of time after excitation with laser pulses of different energies. At $8 \mu \mathrm{J} / \mathrm{pulse}(45 \mathrm{~mJ}$ $\mathrm{cm}^{-2} /$ pulse), the particle reaches a maximum expansion of $1.7 \%$ after approximately $15 \mathrm{~ns}$ and 
then contracts again to its original length within $100 \mathrm{~ns}$. At 15 and $20 \mu \mathrm{J} /$ pulse (85 and $115 \mathrm{~mJ}$ $\mathrm{cm}^{-2} /$ pulse, respectively), the particle starts expanding at the same time scale (15 ns) and with the same expansion speed as with $8 \mu \mathrm{J}$ pulses but the expansion process continues until at 100 ns the maximum length is reached. With $20 \mu \mathrm{J}$ pulses, a saturation expansion of almost $5 \%$ is reached. At high pulse energies (here visible at 15 and $20 \mu \mathrm{J} /$ pulse), the particle appears unstable with oscillating length which stabilizes after a few hundred nanoseconds. The length then remains temporarily unchanged until the particle contracts after $5 \mu \mathrm{s}(15 \mu \mathrm{J})$ or $100 \mu \mathrm{s}(20$ $\mu \mathrm{J})$. The initial length is reached again after $0.1-1 \mathrm{~ms}$. The overall behavior is reproducible from one nanoparticle to another, with minimal variations in the expansion process due to different substrate-particle interfaces. No effects of fatigue were observed when the measurements were repeated (each length measurement consists of 1200 expansion-contraction cycles). 

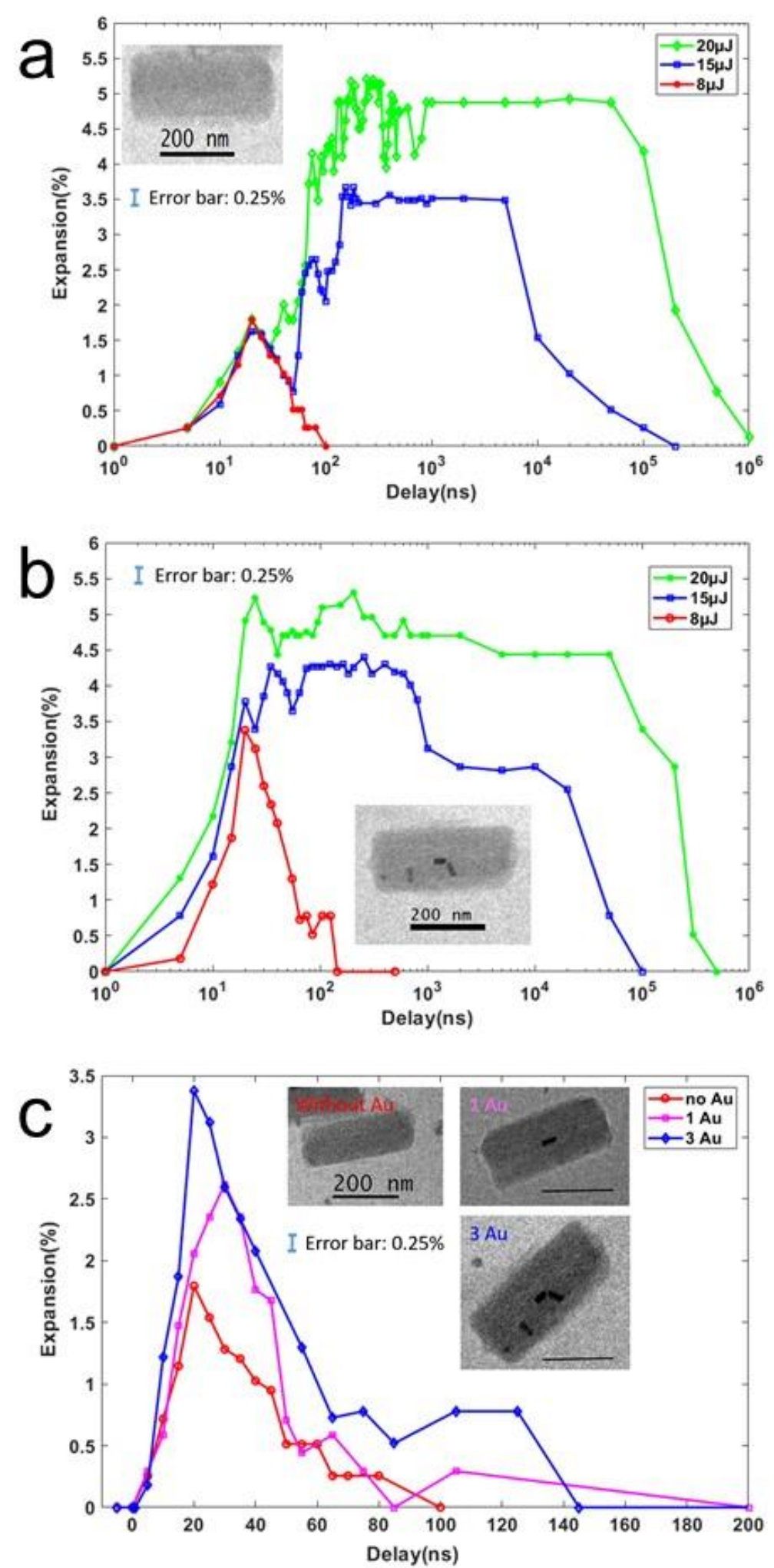

Figure 3: Expansion of an SCO particle as a function of time after an IR pulse. (a) shows a pure SCO particle (length $390 \mathrm{~nm}$ ) and (b) shows a SCO particle (390 nm) encapsulating 3 gold nanorods. The results are shown for different pulse energies. All SCO particles were on 
the same TEM grid. c) Expansion of a pure SCO particle (310 x $190 \mathrm{~nm})$, a SCO particle (370 $x 160 \mathrm{~nm})$ filled with one gold nanorod and, a SCO particle $(390 \times 160 \mathrm{~nm})$ with three gold nanorods. The laser power was always $45 \mathrm{~mJ} \mathrm{~cm}^{-2} /$ pulse. The insets show the respective particles where the measurements were undertaken. Another example is shown in Figure SI3.

Figure 3b shows the behaviour of a SCO particle encapsulating three Au nanorods. For pulse energies where no saturation in pure SCO is reached, less laser power is needed to achieve the same length changes as in unfilled SCO. For example, $8 \mu \mathrm{J}$ pulses lead to $1.7 \%$ expansion in pure $\mathrm{SCO}$ and $3.5 \%$ expansion when three Au rods are encapsulated. Furthermore, the maximum expansion at higher pulse energies is reached earlier than in pure SCO. Whereas the maximum expansion at $20 \mu \mathrm{J}$ pulses in pure SCO appears after approximately $100 \mathrm{~ns}$ (Figure 3a), the same expansion is already reached after $25 \mathrm{~ns}$ if the SCO particle encapsulates three gold nanorods (Figure 3b).

The effect of different numbers of Au particles inside one SCO particle on the expansion is compared in Figure 3c for a pure SCO particle, a SCO particle with one Au nanorod and a particle with three Au nanorods, all exposed to the same pulse energy $(8 \mu \mathrm{J})$. The more Au rods are embedded, the higher and faster the expansion is up to saturation.

\subsection{Time-resolved study on assembly of nanoparticles by optical spectroscopy}

The optical absorbance of a macroscopic sample in the 400 to $800 \mathrm{~nm}$ range (Figure SI4) is steadily decreasing as the wavelength increases and it is higher in the LS state. The absorbance of the Au@SCO exhibits two absorption peaks. The first one around $520 \mathrm{~nm}$ is related to the transverse plasmon resonance of the Au nanorods. The second marked one appears in the 650 to $850 \mathrm{~nm}$ spectral range. It is associated to the longitudinal plasmon resonance of the $\mathrm{Au}$ nanorods. The maximum of the absorption of Au@SCO is centered around $740 \mathrm{~nm}$ when the 
SCO compound is in the LS state and is shifted towards $705 \mathrm{~nm}$ when the SCO compound is brought to the HS state. We have previously shown that this shift is related to change of the index of refraction of the SCO compound upon the LS to HS transition ${ }^{[41]}$.

Figure 4a displays the typical increase of the transmission of an Au@ SCO at $\mathrm{T}=333 \mathrm{~K}$ upon excitation by a nanosecond laser pulse at $520 \mathrm{~nm}$ and with a fluence of $\sim 34 \mathrm{~mJ} \mathrm{~cm}^{-2}$ (green dots). At this temperature the sample is just below the descending branch of the thermal hysteresis loop. Upon excitation, the transmission rapidly increases within few tens of nanoseconds, remains almost constant during almost $20 \mu$ s and then decreases and recovers its initial state after about $1 \mathrm{~ms}$. The increase of the transmission $\mathrm{T}(\mathrm{t})$ is fitted using the following function $\mathrm{T}(\mathrm{t})=\Delta \mathrm{T}\left(1-\exp \left(-\mathrm{t} / \mathrm{t}_{\mathrm{s}}\right)\right)$ where $\Delta \mathrm{T}$ and $\mathrm{t}_{\mathrm{s}}$ are the change in transmission and switching time, respectively. Similarly after the plateau, the relaxation of the transmission of the sample towards its initial value is fitted using the following decay law $T(t)=\Delta T \exp \left(-t / t_{r}\right)$ where $t_{r}$ is the relaxation time. As displayed in Figures $4 \mathbf{a}$ the data are well fitted considering $\mathrm{t}_{\mathrm{s}}=18 \pm 2$ ns and $t_{r}=410 \pm 50 \mu \mathrm{s}$. Besides, the change in amplitude of the transmission indicates that about $45 \%$ (i.e. the HS fraction $\gamma_{\mathrm{HS}} \approx 45 \%$ ) of the sample is switched from the LS to HS state (see experimental section).

The same experiment was performed at $9.5 \mathrm{~mJ} \mathrm{~cm}^{-2}$ and $19 \mathrm{~mJ} \mathrm{~cm}^{-2}$ laser fluences, as displayed in Figure 4a (blue and red dots). For the lowest laser fluence the switching time increases to $\sim 250 \pm 50 \mathrm{~ns}$, the plateau lasts almost the same while the relaxation rate decreases: $\mathrm{t}_{\mathrm{r}}=120 \pm 20 \mu \mathrm{s}$ and $70 \pm 10 \mu \mathrm{s}$ for $19 \mathrm{~mJ} \mathrm{~cm}^{-2}$ and $9.5 \mathrm{~mJ} \mathrm{~cm}^{-2}$ laser fluences, respectively.

The same experiment was repeated with a different wavelength of $470 \mathrm{~nm}$, with and without embedded gold nanoparticles (Figure 4b). The difference between SCO and Au@SCO is marked. The change in $\gamma_{\mathrm{HS}} \sim 5 \%$ for the SCO sample is lesser compared to the change recorded for Au@SCO $\left(\gamma_{\mathrm{HS}} \sim 10 \%\right)$. Hence, the relaxation time recorded for the $\mathrm{SCO}\left(\mathrm{t}_{\mathrm{r}}=12.5 \pm 1 \mu \mathrm{s}\right)$ is faster than the one for the Au@SCO $\left(t_{r}=65 \pm 5 \mu \mathrm{s}\right)$. Let us notice that upon irradiation in the 
SPR band we readily damaged the sample after few nanosecond laser shots, even with the lowest accessible photo-switching fluence $\left(\sim 5 \mathrm{~mJ} \mathrm{~cm}^{-2}\right)$, due to a too intense local heating of the SCO at the interface with the gold nanorods.

(a)

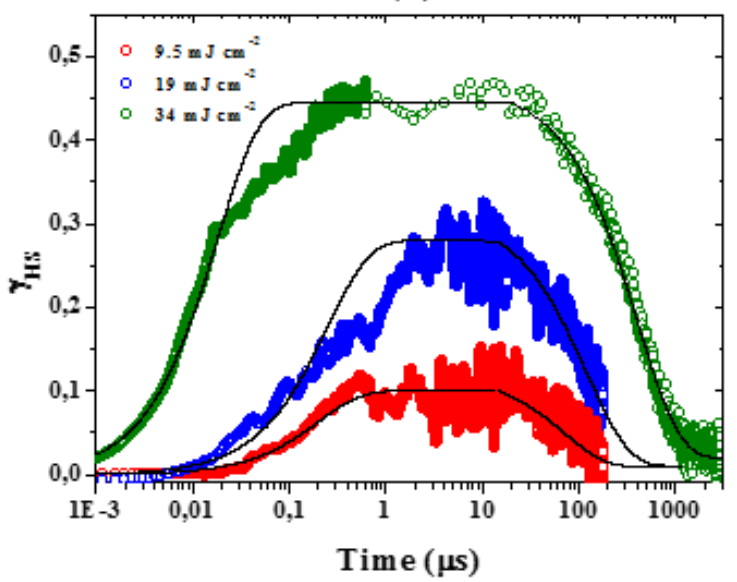

(b)

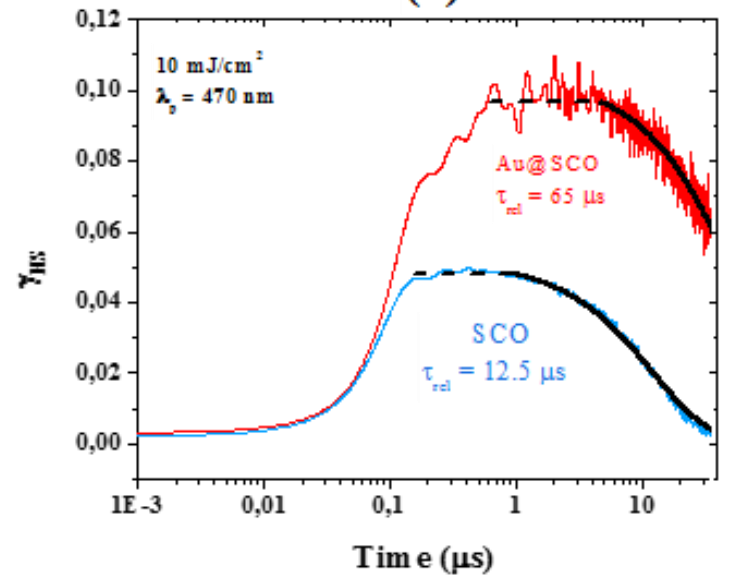

Figure 4: (a) Photoswitching kinetics of Au@SCO upon excitation by nanosecond laser pulses at 520 nm for various laser fluences. (b) Photoswitching kinetics of Au@SCO and SCO upon

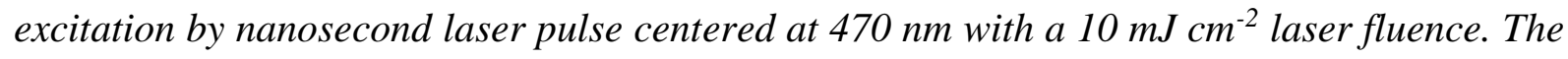
solid lines are fits of $\gamma_{H S}(t)$ using the equations described in the text.

\subsection{Discussion}

We have previously proposed a possible mechanism accounting for the LS to HS state photoswitching of a macroscopic SCO sample, displaying a broad thermal hysteresis loop, upon excitation by nanosecond laser pulses ${ }^{[32,30]}$. The proposed scenario makes it possible to describe the evolution of the sample in the $\left(\mathrm{T}, \gamma_{\mathrm{HS}}\right)$ phase diagram upon excitation as displayed in Figure 5. The sample is initially in the LS state $\left(\gamma_{\mathrm{HS}}=0\right)$ near the ascending branch of the thermal hysteresis loop (point A). Upon excitation and absorption of the laser light by the sample or the substrate, the temperature rapidly increases within the sample while most of the SCO remains in the LS state (Point B). When the fluence is high enough, the SCO sample is over heated and the HS fraction rapidly growths. Then as heat diffuses, the average temperature lowers due to 
the endothermic nature of the LS to HS transition process. This process stops when the temperature of the sample reached the ascending branch of the thermal hysteresis loop (Point C). Beyond this temperature, the HS state fraction remains constant whereas the temperature further decreases. This lasts as long as the temperature of the sample has not reached the descending branch of the thermal hysteresis loop (Point D). Below that latter temperature both the LS fraction and temperature relax back to their initial position within the (T, $\left.\gamma_{L S}\right)$ phase diagram.

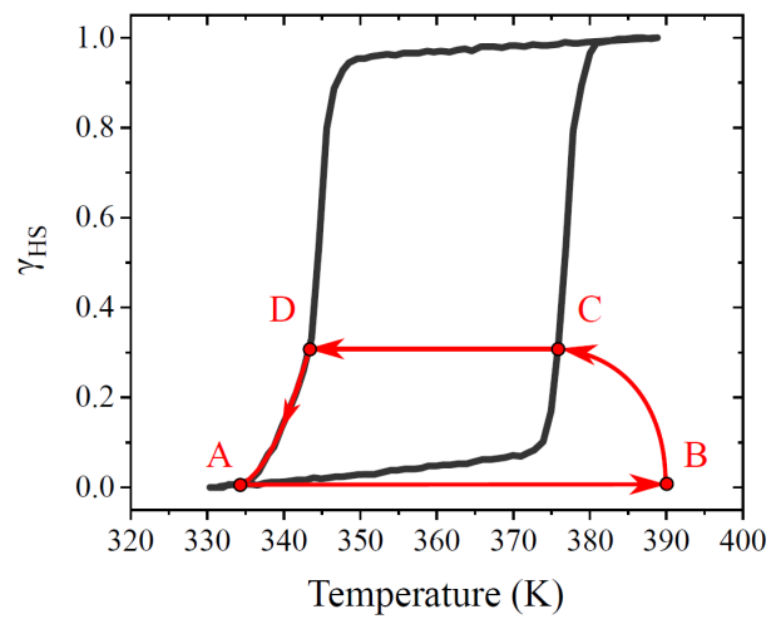

Figure 5: Evolution of the sample within the thermal hysteresis loop under a heat pulse.

The time-resolved optical measurements follow this model quite well. In such experiments, light is directly absorbed by the sample (made of an ensemble of nanoparticles). The temperature increase inside such sample, $\Delta \mathrm{T}$, can be written as:

$$
\Delta \mathrm{T}=\alpha\left(\mathrm{l}_{\mathrm{p}}\right) \mathrm{E}_{\mathrm{p}} /\left(\mathrm{e} \rho \mathrm{C}_{\mathrm{p}}\right)
$$

where $\rho, \alpha\left(l_{p}\right), E_{p}, e, C_{p}$ are the density of SCO particles, absorption at the pump wavelength $1_{\mathrm{p}}$, the laser fluence, the thickness, and the heat capacity of the sample, respectively. The latter physical parameters are known for SCO samples. However, it is noteworthy that due to the sample preparation and the random packing of the nanoparticles, the actual density $\rho$ of our sample is somehow uncertain. We also suppose that, apart from the absorption, the physical 
parameters of SCO and Au@SCO are almost similar. Finally, we consider that $60 \%$ of laser fluence $E_{p}$ is absorbed by the $4 \mu \mathrm{m}$ thick sample (composed of SCO nanoparticles, see experimental section) with $\rho=0.9 \mathrm{~g} \mathrm{~cm}^{-3}, \mathrm{C}_{\mathrm{p}}=1.0 \mathrm{~J} \mathrm{~g}^{-1} \mathrm{~K}^{-1}$ and $\mathrm{e}=4 \mu \mathrm{m}$. As a general consequence, the speed at which HS fraction grows and its amount depend on the amplitude of the overheating $\Delta \mathrm{T}$ : the higher the laser fluence, the higher $\Delta \mathrm{T}$ and the faster the switching time. For $\mathrm{E}_{\mathrm{p}}=34 \mathrm{~mJ} \mathrm{~cm} \mathrm{~cm}^{-2}$ pump fluence, one readily finds that $\Delta \mathrm{T} \sim 56 \mathrm{~K}$. According to this estimation and knowing the initial temperature of the sample $\mathrm{T}_{0}=333 \mathrm{~K}$, the temperature of the sample upon excitation is $\mathrm{T}_{0}+\Delta \mathrm{T}=389 \mathrm{~K}$. Thus, based on the $\left(\mathrm{T}, \gamma_{\mathrm{HS}}\right)$ phase diagram (Figure 5), the sample remains in the HS state when it cools down right after the laser pulse. Hence, upon excitation, the HS fraction increases. After this rapid increase $\gamma_{\mathrm{HS}}$ remains almost constant for $\sim 20 \mu$ s and then the sample relaxes back to its initial LS state. If the laser fluence is reduced to $20 \mathrm{~mJ} \mathrm{~cm}^{-2}, \Delta \mathrm{T} \sim 33 \mathrm{~K}$ (from (1)) and the sample is just brought about the ascending branch of the thermal hysteresis loop. The increase in the HS fraction $\gamma_{\mathrm{HS}}$ is lesser and happens on a longer time scale. In Figure $4 \mathbf{a}$ for laser fluence of $9.5 \mathrm{~mJ} \mathrm{~cm}^{-2}$ the increase of $\gamma_{\mathrm{HS}}$ is small $(\sim 0.05 \%)$ but, according to the calculated increase in temperature, it is higher than expected. This may result from the fact that our simple computation neglects firstly that, due to the high absorption of the sample (thickness $4 \mu \mathrm{m}$ ), particles at the top of the samples absorb more strongly than those at the bottom and secondly, within the excited area part of the Au@SCO nanohybrids may contain more than one gold nanorods (as seen on the TEM pictures, Figure 2).

Equation 1 also indicates that $\Delta \mathrm{T}$ and therefore $\gamma_{\mathrm{HS}}$ depends on the absorption coefficient of the sample. If one tunes the laser wavelength towards a spectral region where the absorption of the samples is higher, the increase in temperature should be higher and $\gamma_{\mathrm{HS}}$ should be increased. This is indeed what we observe as we tune the laser wavelength from $520 \mathrm{~nm}$ to $470 \mathrm{~nm}$, the laser fluence for which a sensible change in $\gamma_{\mathrm{HS}}$ is largely reduced (see Figure SI4). Moreover, as the Au@SCO NPs have a stronger absorption than SCO NPs, due to the inclusion of Au 
nanorods, $\gamma_{\mathrm{HS}}$ is higher in Au@SCO NPs upon excitation compared to SCO NPs. All these conclusions are in agreement with the data presented in Figure 4.

Compared to the optical measurements, the heating processes during the UTEM measurements are different. Indeed, based on an earlier analysis of TEM measurements ${ }^{[45,46]}$, we have to assume that heating of the pure SCO particles occurs mainly through the substrate. This is due to the low absorption of SCO at the used excitation wavelength (1064 nm) for these experiments. Heating of the substrate occurs by absorption of the $\mathrm{Cu}$ grid and to a smaller extent by the graphene film. The high thermal conductivity of the graphene film ensures rapid heat conduction to the particles. The heating of the pure SCO particles is therefore mainly limited by the interfacial area with the graphene which is difficult to control and varies slightly between different particles. This finally determines the timescale of heating and expansion of SCO. Indeed, a variation in the onset of heating between different samples was observed in some cases. However, SCO particles encapsulating Au rods always show rapid heating at all laser powers within approximately $20 \mathrm{~ns}$ (Figures 3b and c). This is understandable due to the more efficient heat transfer from the Au rods that are embedded in the particles and share a large interface with SCO. This demonstrates that the plasmonic heating effect is indispensable if rapid switching of devices at low energies of the laser pulses is desired.

The temporal development of the length of pure (unfilled) SCO particles after laser pulses shows that the onset of expansion occurs approximately $10 \mathrm{~ns}$ after the laser pulse, irrespective of the pulse energy. From the left slope of the curves, an averaged expansion speed for these particles $(390 \mathrm{~nm}$ ) of approximately $0.4 \mathrm{~m} / \mathrm{s}$ is obtained. Towards the maximum expansion of $4.5-5 \%$, which is reached at higher pulse energies, the average expansion speed is slightly less.

The comparison with gold-filled particles (Figure 3b) shows that the expansion for lowenergy pulses ( 8 or $15 \mu \mathrm{J})$ is higher at the same pulse energies if gold is encapsulated. Since the major contribution from the $\mathrm{Au}$ particles is plasmonic heating, the influence on the expansion of SCO has to be of purely thermal nature. It is obvious that an increasing number of $\mathrm{Au}$ 
particles has more influence on heating (Figure 3c). Due to the more efficient heat transfer, the expansion is faster than if heat has to be transported via the substrate. However, once the particles expand, the expansion speed is almost the same in pure SCO and Au@SCO in the lower expansion regime. The maximum expansion of Au@SCO is reached here after 20-30 ns (Figure 3), while more than $100 \mathrm{~ns}$ are needed for pure SCO.

For high excitation energies, the photoswitching time scale, the plateau reached after the expansion and the relaxation rates to the initial LS state agree well with the model proposed from the time-resolved optical measurements (equation 1 and Figure 5). However, this is not the case for lower excitation energies and this probably comes from different heating mechanisms.

Equation 1 holds for an ensemble or a single SCO or Au@SCO NPs exhibiting a spin state transition associated with a thermal hysteresis loop. In order to discuss the thermal evolution of the system, we use a mechanoelastic model, which was previously applied to simulate the dynamic phenomena after ultrafast photoexcitation ${ }^{[24]}$. In this model, the molecules, represented as rigid spheres, are situated in open boundary lattices and the elastic interactions are mimicked by springs linking a molecule to its closest neighbours. When a molecule changes its state, its volume varies, which results in an elongation or a compression first of its closest springs, which determines the change in position of molecules and a propagation of the initial perturbation.

The initial evolution of the system was simulated (a) by randomly creating an additional given number of HS molecules (50\% in our case), whose temperature is increased by $\Delta \mathrm{T}=300$ $\mathrm{K}$ to mimic a direct absorption of light by the compound; (b) by the heat diffusion from a hot substrate towards the sample, or (c) by heat diffusion from 5 hot gold particles inside the lattice.

The dynamics of the "temperatures" of the system is then phenomenologically described for every molecule $i$ by the following differential equation (2).

$$
\frac{\mathrm{dT}_{\mathrm{L}}^{\mathrm{i}}}{\mathrm{dt}}=-\alpha \cdot\left(\mathrm{T}^{\mathrm{i}}-\left\langle\mathrm{T}_{\mathrm{ij}}\right\rangle\right)-\beta \cdot\left(\mathrm{T}_{\text {edge }}^{\mathrm{i}}-\mathrm{T}_{\mathrm{B}}\right)
$$


where $T^{i}$ is the temperature corresponding to the $\mathrm{i}$ molecule, $\left\langle T_{i j}\right\rangle$ is the average temperature of all neighbors of the $\mathrm{i}$ molecule, $\alpha$ is the diffusion coefficient between molecules, $T_{B}$ is the thermal bath temperature, $\mathrm{T}_{\text {edge }}^{\mathrm{i}}$ is the temperature of molecules in contact with the thermal bath, and $\beta$ is the heat transfer coefficient to the bath.

In Figure 6 we present the evolution of the temperatures in the system for all three cases presented above, using equation (2), for a system composed of 10,000 molecules. In all these temperature maps, one can clearly see the inhomogeneity of the temperature inside the sample. For initially randomly switched molecules (Figure 6 left panel), as the nucleation centers are close to each other, the temperatures of all molecules in the system vary immediately after the first step following the switching as the heat propagates in all directions (from a to c). A hot substrate (Figure 6 middle panel) divides the sample in two parts, separated by a moving interface (from d to e): one hot and the other one maintaining its initial temperature for a longer time. Finally, the five gold particles (Figure 6 right panel) act as nucleation centers, changing step by step the temperatures of neighboring sites, while further sites keep their temperature for a while (from $\mathrm{f}$ to $\mathrm{g}$ ). This creates large variety of behaviors based on out-of-equilibrium inhomogeneous heating. The lower the switched HS fraction, the higher the inhomogeneity of temperatures inside the lattice. This is most likely at the origin of the difference between the optical measurements where a plateau in the photoswitching profile is observed and the UTEM measurements where this plateau only appears above $60 \%$ of photoswitching. It indicates that uniform heating throughout the whole particle is necessary to lead to the appearance of a hysteresis. Short and localized heating, as it appears when heating occurs from the substrate or from few localized heating centers such as the metal particles, does not lead to collaborative expansion of the whole particle and therefore not to a hysteresis when the saturation expansion is not reached. 

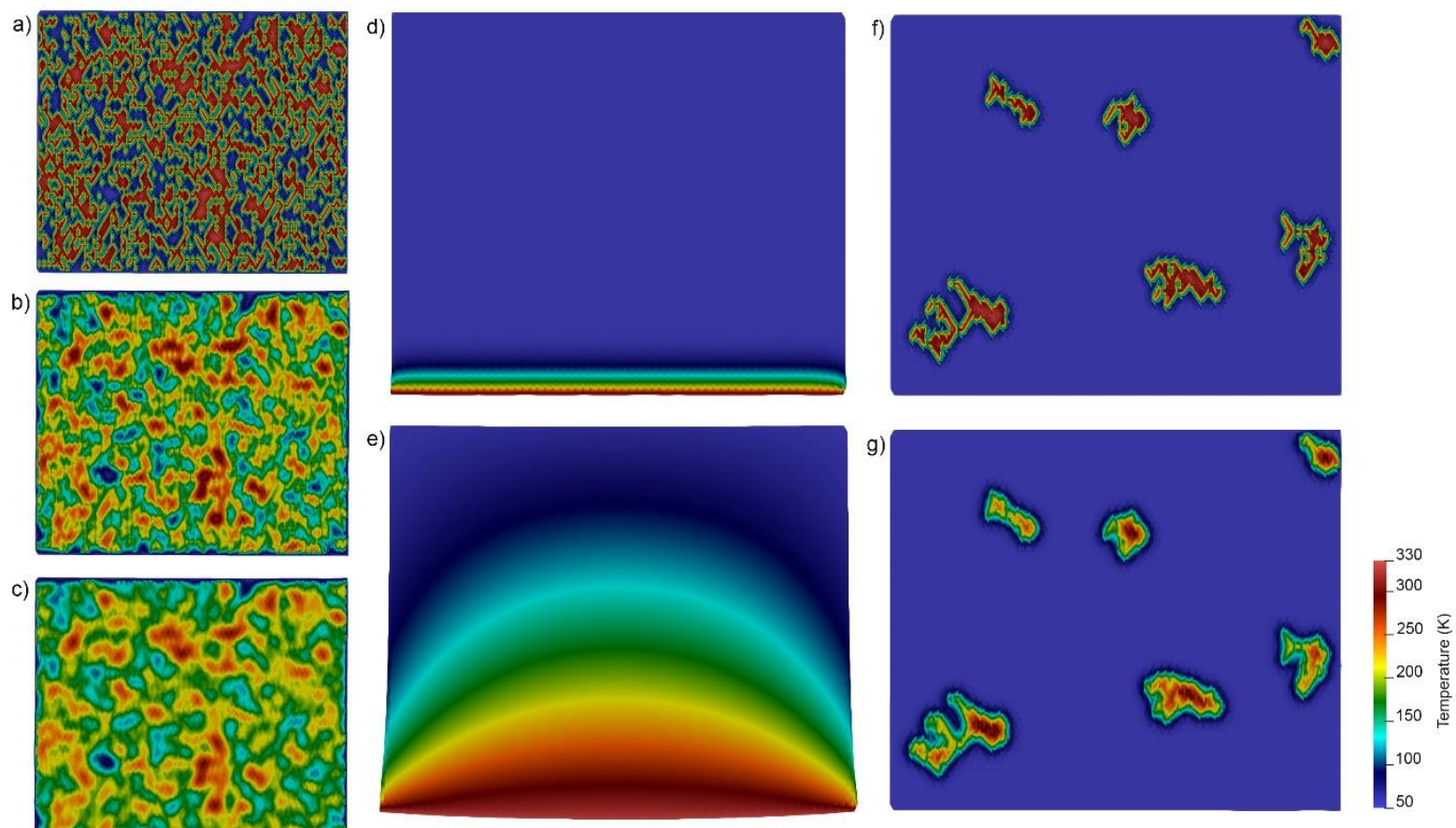

Figure 6: Evolution of temperatures inside the system upon heat diffusion from half of the molecules randomly switched (left panel from a to c), heat diffusion from a hot substrate (middle panel from d to e), heat diffusion from six hot gold particles (right panel, from $f$ to $g$ ).

\section{Conclusion}

In this study, we have investigated the behavior of spin crossover nanoparticles, embedding from zero to seven plasmonically active gold nanorods. TEM measurements performed on single nanoparticles have evidenced the cooperative character of the SCO of such nanocrystals by probing the length change upon temperature variations. This strongly confirms that the breathing of the crystallographic unit cell is reflected at the nanoscopic scale. Moreover, by measuring this length change using UTEM allowed us to probe the photoswitching dynamics of a single nanoparticle and how it is influenced by the presence of gold nanorods. An increasing number of gold nanorods fastens the photoswitching rate from more than 100 ns without any gold particle to $10 \mathrm{~ns}$ with five gold nanorods. This study confirms that heating by laser pulses and heat diffusion inside the particle are the key factors controlling this photoswitching. We evidenced here the efficient plasmonic heating at the single nanoparticle level. We went a step 
further in comparison to most of the current studies on nanoparticles by studying the behavior of individual nano-objects. Moreover, this study also shows that the time-resolved optical study performed on an assembly of nanoparticles is in accordance with the UTEM measurements on individual nanoparticles, leading to similar photoswitching time scales, despite the differences in the heat absorption processes. This is crucial when fast opto-mechanical switches have to be realized with several nanoparticles integrated into a device. It is obvious that plasmonic heating by embedded gold particles leads to the most efficient and fastest switching behaviour and would therefore be adequate for building an optimized and reliable device. A characterization technique combining high spatial and temporal resolution such as UTEM is indispensable when the behaviour of individual SCO nanocrystals needs to be monitored. The combination with optical measurements that average over many particles, gives a comprehensive picture of the processes at different scales. Such an investigation is not only of importance for the understanding of spin crossover phenomena but also for applications of breathing or mechanically active materials (photochromic materials, batteries upon charge and discharge, actuators, nanomachines...).

\section{Experimental Section/Methods}

Ultrafast transmission electron microscopy (UTEM, see Figure SI1) allowed us to observe the behavior of individual particles. In a pump-probe approach ${ }^{[52,53]}$, a pulsed nanosecond laser (1064 nm, $7 \mathrm{~ns}$ pulse duration) is aligned onto a spot $(150 \mu \mathrm{m})$ on the specimen grid in the column of the UTEM. Although the absorption of SCO at $1064 \mathrm{~nm}$ is very low, length changes of pure SCO particles up to saturation could be achieved through heat conduction from the graphene substrate ${ }^{[46]}$ by pulse energies in the range $4-40 \mu \mathrm{J} /$ pulse, corresponding to $20-200$ $\mathrm{mJ} / \mathrm{cm}^{2}$ per pulse. Each IR pulse is followed after an adjustable delay by a $7 \mathrm{~ns}$ electron pulse ${ }^{[52]}$ that served for imaging the particles so that their length could be measured as a function of time. The temporal resolution in this configuration is approximately $10 \mathrm{~ns}$; however, relative 
length changes can be recorded with slightly smaller steps. Due to repulsive electron-electron interactions within the intense pulses which cause a loss of coherence and increased energy width, the intensity of the electron pulses had to be limited ${ }^{[52,54]}$. The pump-probe delay is adjusted by synchronizing the two lasers with an electronic delay unit. Since the length changes of the SCO particles are reversible, the experiment is carried out repeatedly for each measurement in a stroboscopic approach with a repetition frequency of $20 \mathrm{~Hz}$. For recording an image, 1200 pulses are summed up within the typical acquisition time of $60 \mathrm{~s}$. All the timeresolved measurements were performed at room temperature.

The SCO particles are deposited on monolayer graphene grids (Agar Ltd.) for TEM studies. To ensure that no undesired radiation damage of SCO by the electron beam occurred, some particles were exposed deliberately to electron irradiation. After a dose exceeding approximately 1000 electrons $/ \mathrm{nm}^{2}$, the thermally induced expansion isn't observable anymore. Therefore, all pump-probe experiments are carried out at much lower electron dose (typically 0.02 electrons $/ \mathrm{nm}^{2}$ per pulse, that is 24 electrons $/ \mathrm{nm}^{2}$ for the recording of an image), where electron beam effects are negligible.

Time-resolved optical measurements: For the time resolved absorption measurements of an assembly of particles, the samples are prepared as follows. A powder of either SCO or Au@SCO nanoparticles is dispersed in ethanol, stirred, drop-casted and dried on a microscope glass slide. Afterwards, the deposited layer with thickness about $4.0 \pm 2.0 \mu \mathrm{m}$ is covered by another microscope glass slide. The set-up we used to measure the time resolved absorption of the sample is displayed in Figure SI5. The SCO and Au@SCO samples are inserted in an oven whose temperature can be controlled $\left( \pm 0.1{ }^{\circ} \mathrm{C}\right)$ in the range from $300 \mathrm{~K}$ to $440 \mathrm{~K}$. It is then excited by the nanosecond pulse slightly focused on beam spot $\Phi$ of $\sim 1.5 \mathrm{~mm}$ in diameter. The nanosecond pulses are provided by an optical parametric oscillator (Continuum OPO Panther EX). The latter delivers nanosecond pulses tunable from $410 \mathrm{~nm}$ to $2700 \mathrm{~nm}$. The pulse duration and energy are about $\sim 6 \mathrm{~ns}$ and $40 \mathrm{~mJ}$, respectively. The transmission of the sample is probed 
using a continuous wave $(\mathrm{CW})$ laser diode delivering few milliwatts at $785 \mathrm{~nm}$ wavelength. The probe beam transmitted by the sample is focused on the entrance slit of a monochromator to transmit the probe wavelength. The signal transmitted by the monochromator is further filtered by a laser-line filter and focused on a high-speed silicon photodiode connected to a 1 $\mathrm{GHz}$ numerical oscilloscope. The oscilloscope is triggered by the nanosecond laser pulse. It is worth mentioning the central wavelength of the laser diode is centered about the maximum absorption of the Au@SCO sample in the LS state. There is a sensible change in absorption when both SCO and Au@SCO samples are switched from the LS to the HS state ${ }^{41}$.

The way we estimated $\gamma_{\mathrm{HS}}$ in Figure 4 is as follows. We increase the temperature of the oven in which the sample is inserted and we recorded the amplitude of the signal recorded by the photodiode placed after the spectrometer when the sample is set in the LS and HS state. We consider the change in amplitude $\Delta \mathrm{V}$ is associated to $\gamma_{\mathrm{HS}}=1$. Then we consider that upon laser excitation the change in $\gamma_{\mathrm{HS}}(\mathrm{t})$ scales with respect to the signal $\mathrm{V}(\mathrm{t})$ recorded by the photodiode: $\gamma_{H S}(\mathrm{t})=\Delta \mathrm{V}(\mathrm{t}) / \Delta \mathrm{V}$

Supporting Information ((delete if not applicable))

Supporting Information is available from the Wiley Online Library or from the author.

\section{Acknowledgements}

CNRS, University of Bordeaux, Nouvelle Aquitaine Region and the ANR program (HEROES ANR-17-CE09-0010-01) are acknowledged for fundings. Funding by the EQUIPEX program of the Agence Nationale de Recherche, contract ANR-11-EQPX-0041 (project UTEM) and the network METSA (Réseau national de plateformes en microscopie électronique en transmission et sonde atomique) is gratefully acknowledged. $\mathrm{Y}$. $\mathrm{Hu}$ acknowledges support from the China Scholarship Council. G.C. would like to thank Guillaume Rogez to have envisioned the possibility of UTEM in the study of SCO nanohybrids. 
Received: ((will be filled in by the editorial staff))

Revised: ((will be filled in by the editorial staff))

Published online: ((will be filled in by the editorial staff))

References 
1 Kahn, O., Molecular Magnetism. VCH Publishers, New York, (1993).

2 P. Gütlich, H.A. Goodwin, (eds) Spin Crossover in Transition Metal Compounds I-III. Top. Curr. Chem., vols. 233-235. Springer, Berlin / Heidelberg, Germany, 2004.

3 M.A. Halcrow, Spin-Crossover Materials Properties and Applications. Wiley, Chichester 2013.

4 P. Guionneau, M. Marchivie, G. Chastanet, Chem. Eur. J. 2021, 27, 1483.

5 M. D. Manrique-Juarez, S. Rat, L. Salmon, G. Molnar, C. M. Quintero, L. Nicu, H. J. Shepherd, A. Bousseksou, Coord. Chem. Rev. 2016, 308, 395.

6 J. Dugay, M. Gimenez-Marquès, W. J. Venstra, R. Torres-Cavanillas, U. N. Sheaombarsing, N. Manca, E. corondao, H. S. J. van der Zant, J. Phys. Chem. C, 2019, 123, 11.

${ }^{7}$ H. J. Shepherd, I. A. Gural'skiy, C. M. Quintero, S. Tricard, L. Salmon, G. Molnar, A. Bousseksou, Nature. Comm. 2013, 2607.

8 F. Prins, M. Monrabal-Capilla, E. A. Osorio, E. Coronado, H. S. J. van der Zant, $A d v$. Mater. 2011, 23, 1545.

9 K. Senthil Kumar, M. Ruben, Angew. Chem. Int. Ed. Engl. 2021, 60, 7502.

10 C. Lefter, R. Tan, S. Tricard, J. Dugay, G. Molnar, L. Salmon, J. Carrey, A. Rotaru, A. Bousskesou, Polyhedron 2015, 102, 434.

11 K. Ridier, A.-C. Bas, Y. Zhang, L. Routaboul, L. Salmon, G. Molnar, C. Berguad, A. Bousseksou, Nature. Comm. 2020, 3611.

12 N. Konstantinov, A. Tauzin, U. Noumbe, D. Dragoe, B. Kundys, H. Majjad, A. Brosseau, M. Lenertz, A. Singh, S. Berciaud, M.-L. Boillot, B. Doudin, T. Mallah, J.-F. Dayen, J. Mater. Chem. C 2021, 9, 2712. 
13 J.-F. Dayen, N. Konstantinov, M. Palluel, N. Daro, M. Soliman, G. Chastanet, B. Doudin, Materials Horizons 2021, 8, 2310.

14 T. Miyamachi, M. Gruber, V. Davesne, M. Bowen, S. Boukari, L. Joly, F. Scheurer, G. Rogez, T. K. Yamada, P. Ohresser, E. Beaurepaire, W. Wulhekel, Nature. Comm. 2012, 3, 938.

15 M. Urdampilleta, C. Ayela, P.-H. Ducrot, D. Rosario-Amorin, A. Mondal, M. Rouzières, P. Dechambenoit, C. Mathonière, F. Mathieu, I. Dufour, R. Clérac, Scientific reports 2018, 8, 8016.

16 M. D. Manrique-Juarez, S. Rat, F. Mathieu, D. Saya, I. Séguy, T. Leïchlé, L. Nicu, L. Salmon, G. Molnar, A. Bousseksou, Appl. Phys. Lett. 2016, 109, 061903.

17 A. Grosjean, N. Daro, S. Pechev, L. Moulet, C. Etrillard, G. Chastanet, P. Guionneau, Eur. J. Inorg. Chem. 2016, 1961.

18 A. Grosjean, N. Daro, S. Pechev, C. Etrillard, G. Chastanet, P. Guionneau, Eur. J. Inorg. Chem. 2018, 429.

19 S. Lakhloufi, E. Tailleur, W. Guo, F. Le Gac, M. Marchivie, M.-H. Lemée-Cailleau, G. Chastanet, P. Guionneau, Crystals 2018, 8, 363.

20 N. Pittala, F. Thétiot, S. Triki, K. Boukheddaden, G. Chastanet, M. Marchivie, Chem. Mater. 2017, 29, 490 .

21 G. Chastanet, M. Lorenc, R. Bertoni, C. Desplanches, C. R. Chim., 2018, 21, 1075.

22 M. Lorenc, J. Hebert, N. Moisan, E. Trzop, M. Servol, M. Buron-Le Cointe, H. Cailleau, M.-L. Boillot, E. Pontecorvo, M. Wulff, S. Koshihara, E. Collet, Phys. Rev. Lett. 2009, 103, 028301.

23 E. Collet, N. Moisan, C. Balde, R. Bertoni, E. Trzop, C. Laulhe, M. Lorenc, M. Servol, H. Cailleau, A. Tissot, M.-L. Boillot, T. Graber, R. Henning, P. Coppens, M. Buron-Le Cointe, Phys. Chem. Chem. Phys. 2012, 14, 6192. 26 
24 R. Bertoni, M. Lorenc, H. Cailleau, A. Tissot, J. Laisney, M.-L. Boillot, L. Stoleriu, A. Stancu, C. Enachescu, E. Collet, Nat. Mater. 2016, 15, 606.

25 M. G. Reeves, E. Tailleur, P. A. Wood, M. Marchivie, G. Chastanet, P. Guionneau, S. Parsons, Chem. Sci. 2021, 12, 1007.

26 E. Freysz, S. Montant, S. Letard, J.-F. Letard, Chem. Phys. Lett. 2004, 394, 318.

27 S. Bonhommeau, G. Molnar, A. Galet, A. Zwick, J.A. Real, J.J. McGarvey, A. Bousseksou, Angew. Chem., Int. Ed. Engl 2005, 44, 2.

28 S. Cobo, D. Ostrovskii, S. Bonhommeau, L. Vendier, G. Molnar, L. Salmon, K. Tanaka, A. Bousseksou, J. Am. Chem. Soc. 2008, 130, 9019.

29 E. Collet, L. Henry, L. Pineiro-Lopez, L. Toupet, J.A. Real, Curr. Inorg. Chem. 2016, 6, 61. 30 O. Fouche, J. Degert, G. Jonusauskas, N. Daro, J.-F. Letard, E. Freysz, Phys. Chem. Chem. Phys. 2010, 12, 3044.

31 M. Castro, O. Roubeau, L. Pineiro-Lopez, J.A. Real, J.A. RodriguezVelamazan, J. Phys. Chem. C 2015, 119, 17334.

32 G. Galle, C. Etrillard, J. Degert, F. Guillaume, J.-F. Letard, E. Freysz, Appl. Phys. Lett. 2013, $102,063302$.

33 F. Guillaume, Y.A. Tobon, S. Bonhommeau, J.-F. Letard, L. Moulet, E. Freysz, Chem. Phys. Lett. 2014, 604, 105.

34 W. Hellel, A. Ould Hamouda, J. Degert, J.-F. Letard, E. Freysz, Appl. Phys. Lett. 2013, 103, 143304;

35 E.Freysz, J.-F.Letard, French Patent, 2012 FR 2993978 eA1

36 L. Salmon, L. Catala, C. R. Chim., 2018, 21, 1.

37 G. Baffou, R. Quidant, Laser Photonics Rev. 2013, 7, 171. 
38 I. Suleimanov, J. Sánchez Costa, G. Molnár, L. Salmon A. Bousseksou, Chem. Commun., $\mathbf{2 0 1 4}, 50,13015$.

39 D. Qiu, L. Gu, X. L. Sun, D. H. Ren, Z. G. Gu, Z. Li, RSC Adv. 2014, 4, 61313.

40 L. Moulet, N. Daro, S. Mornet, N. Vilar-Vidal, G. Chastanet P. Guionneau, Chem. Commun., 2016, 52, 13213.

41 M. Palluel, N. M. Tran, N. Daro, S. Buffière, S. Mornet, E. Freysz G. Chastanet, G. Adv. Funct. Mater. 2020, 30, 2000447.

42 R. Sanchis-Gual, R. Torres-Cavanillas, M. Coronado-Puchau, M. Gimenez-Marques, E. Coronado, J. Mater. Chem. C. 2021, 9, 10811.

43 R. Torres-Cavanillas, R. Sanchis-Gual, J. Dugay, M. Coronado-Puchau, M. GimenezMarques, E. Coronado, Adv. Mater. 2019, 31, 1900039.

${ }^{44}$ S. Liu, K. zhou, T. Yuan, W. Lei, H.-Y., Chen, X. Wang, W. Wang, J. Am. Chem. Soc., 2020, 142,15852 .

45 R. M. Van der Veen, O.-H. Kwon, A. Tissot, A. Hauser, A. H. Zewail, Nature. Chem. 2013, 5,395 .

46 S. T. Park, R. M. van der Veen, Struc. Dyn. 2017, 4, 044028.

47 O. Roubeau, Chem. - A Eur. J., 2012, 18, 15230.

48 J. Kröber, J.-P. Audière, R. Claude, E. Codjovi, O. Kahn, J.G. Haasnoot, F. Grolière, C. Jay, A. Bousseksou, J. Linarès, F. Varret, A. Gonthier-Vassal, Chem. Mater. 1994, 6, 1404.

49 A. Michalowicz, J. Moscovici, B. Ducourant, D. Cracco O. Kahn, Chem. Mater. 1995, 7, 1833.

50 L. Moulet, N. Daro, C. Etrillard, J.-F. Létard, A. Grosjean P. Guionneau, Magnetochemistry, 2016, 2, 10. 
51 A. Grosjean, P. Négrier, P. Bordet, C. Etrillard, D. Mondieg, S. Pechev, E. Lebraud, J.-F. Létard, P. Guionneau, Eur. J. Inorg. Chem. 2013, 796.

52 M. Picher, K. Bücker, T. LaGrange F. Banhart, Ultramicroscopy 2018, 188, 41.

53 K. Bücker, M; Picher, O. Crégut, T. LaGrange, B. W. Reed, S. T. Park, D. J. Masiel, F. Banhart, Ultramicroscopy 2016, 171, 8.

54 S. K. Sinha, A. Khammari, M. Picher, F. Roulland, N. Viart, T. LaGrange, F. Banhart, Nature Commun. 2019, 10, 3648. 